\title{
Pharmacological Management of Atrial Fibrillation: One, None, One Hundred Thousand
}

\author{
Fabiana Lucà, ${ }^{1,2,3}$ Mark La Meir, ${ }^{2}$ Carmelo Massimiliano Rao, ${ }^{1}$ Orlando Parise, ${ }^{1}$ \\ Ludovico Vasquez, ${ }^{3}$ Rocco Carella, ${ }^{1}$ Roberto Lorusso, ${ }^{4}$ Benedetto Daniela, ${ }^{1}$ Jos Maessen, ${ }^{2}$ \\ Gian Franco Gensini, ${ }^{1}$ and Sandro Gelsomino ${ }^{1,2}$
}

${ }^{1}$ Department of Heart and Vessels, Careggi Hospital, Viale Morgagni 85, 50134 Florence, Italy

${ }^{2}$ Department of Cardiothoracic Surgery, Academic Hospital, 6202 AZ Maastricht, The Netherlands

${ }^{3}$ Cardiology Unit, Barone Romeo Hospital, 98066 Patti Messina, Italy

${ }^{4}$ Cardiac Surgery Units, Community Hospital, 25128 Brescia, Italy

Correspondence should be addressed to Sandro Gelsomino, sandro.gelsomino@libero.it

Received 2 January 2011; Accepted 21 February 2011

Academic Editor: Jalal K. Ghali

Copyright () 2011 Fabiana Lucà et al. This is an open access article distributed under the Creative Commons Attribution License, which permits unrestricted use, distribution, and reproduction in any medium, provided the original work is properly cited.

\begin{abstract}
AF) is associated with a significant burden of morbidity and increased risk of mortality. Antiarrhythmic drug therapy remains a cornerstone to restore and maintain sinus rhythm for patients with paroxysmal and persistent AF based on current guidelines. However, conventional drugs have limited efficacy, present problematic risks of proarrhythmia and cause significant noncardiac organ toxicity. Thus, inadequacies in current therapies for atrial fibrillation have made new drug development crucial. New antiarrhythmic drugs and new anticoagulant agents have changed the current management of AF. This paper summarizes the available evidence regarding the efficacy of medications used for acute management of AF, rhythm and ventricular rate control, and stroke prevention in patients with atrial fibrillation and focuses on the current pharmacological agents.
\end{abstract}

\section{Introduction}

Atrial fibrillation (AF) is the most common cardiac rhythm disturbance seen in clinical practice accounting for approximately one-third of hospitalizations [1]. AF may occur isolated or in association with structural heart disease, contributing substantially to cardiac morbidity and mortality. The estimated prevalence of $\mathrm{AF}$ is $0.4-1 \%$ in the general population, increasing with age $[2,3]$, and it is associated with an higher long-term risk of stroke, heart failure, and allcause mortality, especially in women $[4,5]$.

Management of patients with AF requires knowledge of its pattern of presentation [6] (first diagnosed, paroxysmal, persistent, long-standing, and permanent AF, Figure 1), underlying conditions, and decisions about restoration and maintenance of sinus rhythm, control of the ventricular rate, and antithrombotic therapy.
Antiarrhythmic drug therapy is the first-line treatment for patients with paroxysmal and persistent AF based on current guidelines [6, 7]. Prevention of AF-related complications relies on antithrombotic therapy, control of ventricular rate, and adequate therapy of concomitant cardiac diseases. However, available drug therapy has major limitations, including incomplete effectiveness, cardiac and extracardiac toxicity and risk of life-threatening proarrhythmic complications (antiarrhythmic agents), and bleeding (anticoagulants) [8-11].

Thus, there is a continuing need for new drugs, device, and ablative approaches to rhythm restoration, and simpler and safer stroke prevention regimens are needed for $\mathrm{AF}$ patients on life-long anticoagulation [12].

This paper summarizes the available evidence regarding the efficacy of medications used for ventricular rate control, 


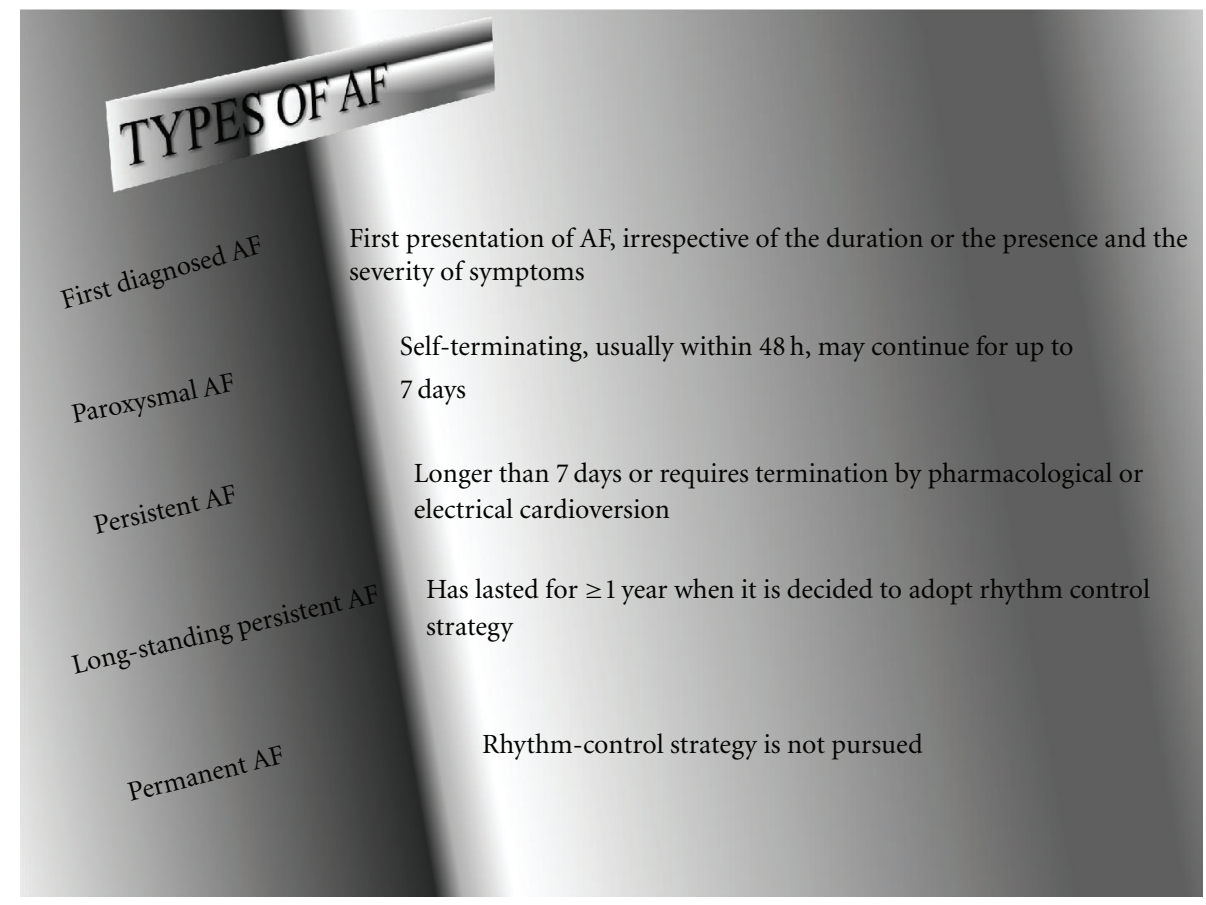

Figure 1: Classification of atrial fibrillation.

stroke prevention, acute conversion, and maintenance of sinus rhythm in patients with atrial fibrillation.

\section{Acute Management}

The acute management of patients with AF is driven by acute protection against thromboembolic events and acute improvement of cardiac function. The severity of AF-related symptoms should drive the decision for acute restoration of sinus rhythm or acute management of the ventricular rate. In stable patients with a rapid ventricular response, the acute control of ventricular rate can be achieved by oral administration of $\beta$-blockers or nondihydropyridine calcium channel antagonists. In contrast, in severely compromised patients, i.v. verapamil or metoprolol may be used [6].

In patients who remain symptomatic despite adequate rate control, or in patients in whom rhythm control therapy is pursued, pharmacological cardioversion of AF may be initiated by a bolus administration of an antiarrhythmic drug (Table 1) [6].

In the acute setting, flecainide has an established effect on restoring sinus rhythm in patients with AF of short duration ( $<24$ hours) $[6,13]$. Patients undergoing flecainide treatment, should be checked for contraindications including structural heart disease, second- or third-degree AV block, left-bundle branch block, right-bundle branch block (when associated with left hemiblock), asymptomatic nonsustained ventricular tachycardia, cardiogenic shock, reduced cardiac output (LVEF < 35\%), post-MI, and significant renal or hepatic impairment $[6,13]$. Flecainide is also a safe and effective agent for termination of AF in patients with WolffParkinson-White (WPW) syndrome [14].
Propafenone is indicated to convert recent onset AF to sinus rhythm in patients without abnormal LV function and ischemia, but it has a limited efficacy to convert atrial flutter $[15,16]$.

In patients with underlying heart disease, amiodarone can be employed as it blocks $\mathrm{Na}^{+}, \mathrm{Ca}^{2+}$, and $\mathrm{K}^{+}$channels [17] and inhibits the consequences of $\alpha$ adrenoceptor and $\beta$-adrenoceptor stimulation [18]. Nonetheless, it does not achieve cardioversion in the short and medium terms [6]. Ibutilide is a useful agent for the pharmacological cardioversion of recent-onset atrial fibrillation but is more effective in terminating atrial flutter. It prolongs the myocardial action potential duration and effective refractory periods in both the atria and the ventricles. The mean times to conversion were $\leq 30$ minutes [19]. Its cellular electrophysiologic mechanism increases the slow inward plateau sodium current and inhibits the outward repolarizing potassium current. Adverse events associated with ibutilide are predominantly proarrhythmic effects [20, 21]. The drug has minimal haemodynamic effects and is associated with few non-cardiovascular adverse events [22-26].

Vernakalant is a relative atrialselective antiarrhythmic agent [27] recently recommended for approval by the European Medicines Agency for rapid cardioversion of recentonset AF to sinus rhythm in adults ( $\leq 7$ days for non-surgical patients; $\leq 3$ days for surgical patients) [28, 29]. Atrial selectivity of vernakalant is achieved by targeting atrialspecific channels: the Kv1.5 channel which carries $\mathrm{K}^{+}$current (IKur) and the Kir3.1/3.4 channel which carries muscarinic $\mathrm{K}^{+}$current (IKAch). Vernakalant can also work to block Ito, late Ina, with minor blockade of IKr currents [30, 31]. 
TABLe 1: Acute rhythm management.

\begin{tabular}{|c|c|c|c|}
\hline Drug & Dose & Followup dose & Risks \\
\hline Amiodarone & $5 \mathrm{mg} / \mathrm{kg}$ i.v. over $1 \mathrm{~h}$ & $50 \mathrm{mg} / \mathrm{h}$ & $\begin{array}{l}\text { Phlebitis, hypotension. Will slow the ventricular rate. } \\
\text { Delayed AF conversion to sinus rhythm. }\end{array}$ \\
\hline Flecainide & $\begin{array}{l}2 \mathrm{mg} / \mathrm{kg} \text { i.v. over } 10 \mathrm{~min} \text {, } \\
\text { or } 200-300 \mathrm{mg} \text { p.o. }\end{array}$ & N/A & $\begin{array}{l}\text { May prolong QRS duration and the QTinterval, and may } \\
\text { increase the ventricular rate due to conversion to atrial } \\
\text { flutter and } 1: 1 \text { conduction to the ventricles. }\end{array}$ \\
\hline Ibutilide & $1 \mathrm{mg}$ i.v. over $10 \mathrm{~min}$ & $\begin{array}{l}1 \mathrm{mg} \text { i.v. over } 10 \mathrm{~min} \\
\text { after waiting for } 10 \mathrm{~min}\end{array}$ & $\begin{array}{l}\text { Can cause prolongation of the QT interval and torsades de } \\
\text { pointes; watch for abnormal T-U waves or QT } \\
\text { prolongation. Will slow the ventricular rate. }\end{array}$ \\
\hline Propafenone & $\begin{array}{l}2 \mathrm{mg} / \mathrm{kg} \text { i.v. over } 10 \mathrm{~min} \text {, } \\
\text { or } 450-600 \mathrm{mg} \text { p.o }\end{array}$ & N/A & $\begin{array}{l}\text { May prolong QRS duration, will slightly slow the } \\
\text { ventricular rate, but may increase the ventricular rate due } \\
\text { to conversion to atrial flutter and } 1: 1 \text { conduction to the } \\
\text { ventricles. }\end{array}$ \\
\hline Vernakalant & $3 \mathrm{mg} / \mathrm{kg}$ i.v. over $10 \mathrm{~min}$ & $\begin{array}{l}\text { Second infusion of } \\
2 \mathrm{mg} / \mathrm{kg} \text { i.v. over } 10 \mathrm{~min} \\
\text { after } 15 \mathrm{~min} \text { rest }\end{array}$ & Recently approved by the European Medicines Agency. \\
\hline
\end{tabular}

A direct comparison with amiodarone in the AVRO trial [32] showed that vernakalant was more effective than amiodarone for the rapid conversion of AF to sinus rhythm (51.7\% versus $5.7 \%$ at $90 \mathrm{~min}$ after the start of treatment, $P<.0001)$. Intravenous vernakalant is generally given at an initial dose of $3 \mathrm{mg} / \mathrm{kg}$, and then an additional $2 \mathrm{mg} / \mathrm{kg}$ if atrial fibrillation conversion fails after $15 \mathrm{~min}$. The elimination half-life is about $2 \mathrm{~h}$. Vernakalant is contraindicated in patients with systolic blood pressure $<100 \mathrm{~mm} \mathrm{Hg}$, severe aortic stenosis, heart failure (class NYHA III and IV), acute coronary syndrome (ACS) within the previous 30 days, or QT interval prolongation [30-33]. Furthermore, before its use, the patients should be adequately hydrated. In addition, ECG and hemodynamic monitoring should be used, and the infusion can be followed by direct current cardioversion (DCC) if necessary [30-33]. The drug is not contraindicated in patients with stable coronary artery disease, hypertensive heart disease, or mild heart failure. The clinical positioning of this drug has not been determined yet, but it is likely to be used for acute termination of recent-onset AF in patients with lone AF or AF associated with hypertension, coronary artery disease, or mild- to moderate- (NYHA class I-II) heart failure [34].

The ACC/AHA/ESC guidelines identify dofetilide, ibutilide, and amiodarone as agents with efficacy for pharmacologic cardioversion of atrial fibrillation $>7$ days and disopyramide, flecainide, procainamide, propafenone, and quinidine as less effective or incompletely studied [13]. The socalled "pill-in-the-pocket" approach may be used in selected, highly symptomatic patients with infrequent (once/month to once/year) recurrence of atrial fibrillation. According to a medium-size trial 1 , oral propafenone $(450-600 \mathrm{mg})$ of flecainide $(200-300 \mathrm{mg}$ ) can be administered by patients safely (1/569 episodes resulting in atrial flutter with rapid conduction) and effectively (94\%, 534/569 episodes) out of hospital. In order to implement the pill-in-the-pocket technique, patients should be screened for indications and contraindications, and the efficacy and safety of oral treatment should be tested in hospital. Finally, patients should be instructed to take flecainide or propafenone when symptoms of AF occur [35].

\section{Long-Term Management}

The restoration and maintenance of sinus rhythm has been shown to be associated with reduced atrial remodeling, improved left ventricular function, reduced symptoms, greater exercise tolerance, increased ability to perform activities of daily living, and improved quality of life [36]. However, rates of attainment and maintenance of sinus rhythm have been suboptimal in comparative studies such as atrial fibrillation followup investigation of rhythm management (AFFIRM) [37], Polish how to treat chronic atrial fibrillation (HOT CAFE) [38], pharmacological intervention in atrial fibrillation (PIAF) [39], rate control versus electrical cardioversion (RACE) [40], strategies of treatment in atrial fibrillation (STAF) [41] and atrial fibrillation and congestive heart failure (AF-CHF) [42]. Furthermore studies failed to demonstrate a survival advantage with either approach by intention-to-treat analysis-both in patients with and without heart failure (HF) $[36,43]$. This is probably because the antiarrhythmic therapies studied had limited efficacy, poor tolerability, and the potential to trigger new arrhythmias. Moreover, several of the antiarrhythmic drugs used for rhythm control in these studies were associated with a significant increase in noncardiovascular deaths [36]. However, the results do not support the hypothesis that rate control is preferable as first-line therapy for AF with respect to survival and do not disprove the hypothesis that maintenance of sinus rhythm is preferable to the continuation of AF, particularly if rate control fails to restore adequate quality of life (QOL) or whether selective approaches are employed. Many posthoc analyses and substudies have assessed QOL, functional status, and exercise tolerance, with the majority demonstrating important benefits associated with achievement of rhythm control. Moreover, some subanalyses and additional trials have suggested that sinus rhythm can be associated with longer survival, including in patients with HF [43]. 
Current guidelines indicate paroxysmal AF is more often managed with a rhythm control strategy, especially if it is symptomatic and there is little or no associated underlying heart disease.

Permanent AF is managed by rate control unless it is deemed possible to restore sinus rhythm when the $\mathrm{AF}$ category is redesignated as "long-standing persistent". Rate control is needed for most patients with AF unless the heart rate during $\mathrm{AF}$ is naturally slow. Rhythm control may be added to rate control if the patient is symptomatic despite adequate rate control, or if a rhythm control strategy is selected because of factors such as the degree of symptoms, younger age, or higher activity levels [6].

\section{Maintenance of Normal Sinus Rhythm}

A number of agents are effective for the maintenance of normal sinus rhythm in patients with atrial fibrillation. According to current guidelines, amiodarone, dronedarone, flecainide, propafenone, sotalol, and disopyramide are recommended for rhythm control depending on the underlying heart disease (Figure 2) [6].

The new ESC 2010 AF guidelines mention for the first time dronedarone as a recommended treatment in patients with AF [6]. Dronedarone is a multichannel blocker which inhibits sodium, potassium, and calcium channels with a noncompetitive antiadrenergic activity. Its short halflife (of approximately $24 \mathrm{~h}$ ) reduces the accumulation in tissues and the low lipophilicity as well as elimination of iodine moieties, which reduces its toxicity. Dronedarone prolongs the action potential duration and reduces heart rate, with low proarrhythmic effect $[1,44]$. Maximum dronedarone plasma concentrations are reached within 1$4 \mathrm{~h}$ [45]. Steady-state concentrations are achieved within 7 days of $400 \mathrm{mg}$ twice daily [46]. Dronedarone is extensively biotransformed by cytochrome P450 (CYP3A4) enzymes, with little excretion of unchanged drug in bile and urine. The elimination half-life (about $24 \mathrm{~h}$ ) is much shorter than that for amiodarone, which is very slow (up to many weeks) because of delayed removal from adipose tissue stores. Potent CYP3A4 inhibitors (e.g., ketoconazole or erythromycin) can raise dronedarone plasma concentrations. Dronedarone increases serum digoxin levels by inhibition of P-glycoprotein intestinal and renal excretion and can raise serum creatinine concentrations by inhibition of renal organic-cation transport [47].

In patients with adrenergic AF and no or minimal structural heart disease, dronedarone is recommended if $\beta$ blocking agents (including sotalol) are not effective [48]. In patients with left ventricular hypertrophy, coronary artery disease, and stable New York Heart Association (NYHA) class $\mathrm{I} / \mathrm{II}$, dronedarone is the antiarrhythmic drug of choice. However, dronedarone should not be used in AF patients with NYHA class III/IV or in unstable patients with NYHA class II. In these patients, $\beta$-blocking agents are recommended as first-line therapy $[6,48]$.

According to 2011 ACCF/AHA/HRS focused update on the management of patients with atrial fibrillation [7], dronedarone is reasonable to decrease the need for hospitalization for cardiovascular events in patients with paroxysmal AF or after conversion of persistent AF. Dronedarone can be initiated during outpatient therapy (Class IIa, Level of Evidence B) [49]. Dronedarone should not be administered to patients with class IV heart failure or patients who have had an episode of decompensated heart failure in the past 4 weeks, especially if they have depressed left ventricular function (left ventricular ejection fraction $\leq 35 \%$, Class IIIHarm, Level of Evidence B) [50].

The EURIDIS (european trial in atrial fibrillation or flutter patients receiving dronedarone for the maintenance of sinus rhythm) and ADONIS (American-Australian-African Trial With Dronedarone in Atrial Fibrillation or Flutter Patients for the Maintenance of Sinus Rhythm) trials [51, 52] found that dronedarone prolongs the time to recurrence AF. Posthoc analyses of pooled EURIDIS and ADONIS data showed that dronedarone greatly decreased the combined endpoint of admission or death. The efficacy of dronedarone for rate control in patients with permanent atrial fibrillation was tested in the ERATO trial [53].

Possessing both rate- and rhythm-control properties, dronedarone has proved safe and effective in preventing $\mathrm{AF}$ recurrence in patients with persistent AF in the DAFNE (dronedarone atrial fibrillation study after electrical cardioversion) trial, the first prospective randomized trial to evaluate its efficacy and safety [54].

In the DIONYSOS [55] (efficacy \& safety of dronedarone versus amiodarone for the maintenance of sinus rhythm in patients with persistent atrial fibrillation), a short-term, randomized, double-blind, parallel-group study, in patients with persistent atrial fibrillation, dronedarone was less efficacious but also less toxic than amiodarone. Recurrence of AF during followup at 12 months and study drug discontinuation occurred in $75 \%$ and $59 \%$ of patients treated with dronedarone and amiodarone, respectively (hazard ratio (HR) 1.59 ; 95\% CI 1.28-1.98; $P<.0001$ ). AF recurrence was more common in the dronedarone arm compared with amiodarone (36.5\% versus $24.3 \%)$. Premature drug discontinuation tended to be less frequent with dronedarone $(10.4 \%$ versus $13.3 \%)$. The safety profile of dronedarone is advantageous in patients without structural heart disease and in stable patients with heart disease. Specifically, dronedarone appears to have a low potential for proarrhythmia. Fewer thyroid, neurologic, dermatologic, and ocular events occurred in the dronedarone group. These data suggest higher tolerability but less efficacy for dronedarone than for amiodarone $[52,55]$.

The ANDROMEDA (antiarrhythmic trial with dronedarone in moderate- to- severe CHF evaluating morbidity decrease) trial in patients in sinus rhythm and systolic left ventricular dysfunction was prematurely discontinued because of increased mortality with dronedarone. The deaths in the dronedarone group were due predominantly to heart failure, and there was no evidence of proarrhythmia or an increased incidence of sudden death. Six months after discontinuation mortality rates were similar, but dronedarone is nevertheless contraindicated in class III and IV heart failure [56]. 


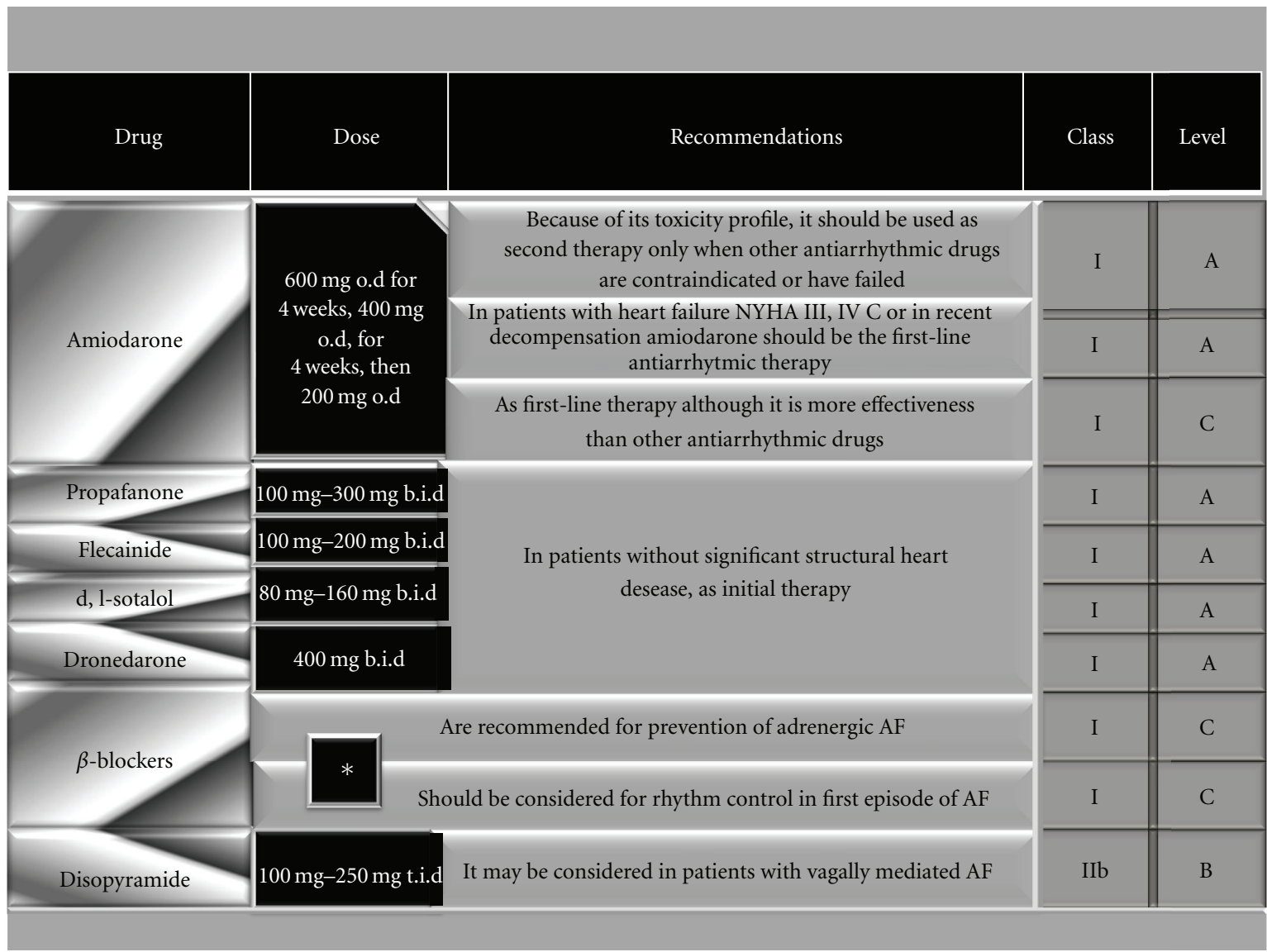

FIgURE 2: ACC/AHA/ESC algorithm for antiarrhythmic maintenance drug therapy. ${ }^{*}$ The dosage must be patient-tailored.

The ATHENA study (A placebo-controlled, doubleblind, parallel arm trial to assess the efficacy of dronedarone $400 \mathrm{mg}$ b.i.d. for the prevention of cardiovascular hospitalization or death from any cause in patients with atrial fibrillation/atrial flutter) randomized 462 patients with paroxysmal or persistent AF or flutter and cardiovascular risk factors to treatment with dronedarone or placebo, assessing a substantially reduction of primary endpoint (all-cause mortality and cardiovascular admissions) $31.9 \%$ versus $39.4 \%$ on placebo, HR $0.76(0.69-0.84)$ driven by reduction in cardiovascular admission events: $29.3 \%$ versus 36.9 on placebo (HR 0.74 (0.67-0.82), but nonsignificant difference in all cause mortality.

Dronedarone improved the composite endpoint of cardiovascular hospitalizations and all-cause mortality in a carefully selected, high-risk, nonpermanent AF population, in addition to its recognized reduction in AF [57]. The rate of cardiovascular mortality was lower in the dronedarone group (2.7\% versus $3.9 \%$; HR $0.71 ; 95 \%$ CI $0.51-0.98)$. The median time to first artial fibrillation or recurrence of atrial flutter was increased by dronedarone, and the likelihood of permanent atrial fibrillation or atrial flutter was greatly reduced [58]. The ATHENA trial excluded patients with decompensated heart failure within the previous 4 weeks, or with NYHA class IV heart failure. There was no evidence of an adverse effect of dronedarone in patient subgroups with a history of congestive heart failure or LV ejection fraction $\leq 35 \%$ [57]. The major adverse cardiac effects of dronedarone are bradycardia and QT prolongation. Torsades de pointes have been reported [57]. Like amiodarone, dronedarone inhibits renal tubular secretion of creatinine, which can increase plasma creatinine levels. However, there is no reduction in glomerular filtration rate. Dronedarone increases digoxin levels 1.7- to 2.5-fold.31 Dronedarone is predominantly metabolized by the liver (CYP3A4) with a half-life of approximately 19 hours. It should not be administered with strong inhibitors of CYP3A4 (e.g., ketoconazole and macrolide antibiotics), because these may potentiate the effects of dronedarone. It can be administered with verapamil or diltiazem, which are moderate CYP3A4 inhibitors, but low doses of these agents should be used initially and titrated according to response and tolerance [59]. Dronedarone does not alter the international normalization ratio when used with warfarin. The recommended oral dose of dronedarone is $400 \mathrm{mg}$ twice a day with meals. An intravenous form is not available.

In maintaining sinus rhythm, amiodarone is more effective than others agent and has restricted proarrhythmic potential, but, because of its very long half-life and profile toxicity with severe extracardiac side effects [60], should 
be generally use when other therapies have failed or are contraindicated. In patients with severe heart failure, NYHA class III and IV or recently unstable NYHA class II (decompensation within the prior month), amiodarone should be the drug of choice [6].

Nonetheless, although amiodarone is the widely considered for maintenance of sinus rhythm in atrial fibrillation management, it lacks FDA approval for this indication.

\section{Inhibitors of the Renin-Angiotensin-Aldosterone System}

Atrial angiotensin II concentrations increase in atrial fibrillation [61] and stimulation of its receptors activates nicotinamide adenine dinucleotide-phosphate (NADPH) oxidase to produce oxidative stress or inflammation [62]. Several studies [63-65] suggest benefit of angiotensin-converting enzyme (ACE) inhibitors or angiotensin II type 1 (AT1)blockers in prevention of atrial fibrillation, especially in patients with left ventricular hypertrophy or dysfunction. particularly, patients treated with amiodarone plus irbesartan showed a lower rate of recurrence of atrial fibrillation than did patients treated with amiodarone alone $[65,66]$.

However, the large placebo-controlled GISSI-AF trial showed that valsartan did not reduce recurrence rates of atrial fibrillation, raising questions about the value of AT1 blockers in secondary prevention [67]. Furthermore, preliminary results of the ACTIVE I trial, including 9016 atrial fibrillation patients during a follow-up of 4.1 years, showed that irbesartan did not prevent cardiovascular events and had no effect on atrial fibrillation burden [68].

Further prospective studies are needed to establish the potential therapeutic value of ACE inhibitors and AT1 blockers in prevention of atrial fibrillation and to define the populations of patients that benefit.

Aldosterone exerts many cardiac effects. In small animals, it causes atrial fibrosis, and spironolactone prevents fibrosis [69]. Selective aldosterone receptor blockade also suppresses atrial fibrillation in animal models of heart failure [70]. Plasma aldosterone concentrations increase in patients with atrial fibrillation [71], and atrial expression of the aldosterone receptor is higher in these patients than in those without the disorder [72]. Furthermore, patients with primary hyperaldosteronism have a 12 -fold greater risk of atrial fibrillation than do controls matched for blood pressure [73]. Hence, blockade of aldosterone receptors could be a therapeutic option for patients with atrial fibrillation, but data from trials are not available.

\section{Anti-Inflammatory Agents}

Glucocorticoids have powerful anti-inflammatory properties and have efficacy against atrial fibrillation in animal [74] and clinical [75] studies although their potential toxicity restricts their value in this disorder. Both statins and omega3 fatty acids have anti-inflammatory and antioxidant actions. Statins are effective against several substrates that maintain atrial fibrillation $[76,77]$. Statins are of benefit in prevention of atrial fibrillation, especially for postoperative AF [78]. Epidemiological data about the effects of omega- 3 fatty-acids on occurrence of AF are conflicting [79]. Animal studies [80] show model dependent atrial-fibrillation-preventing effects, suggesting that omega-3 fatty acids prevent atrial fibrillation, especially in patients at risk of fibrotic structural remodeling. Peroxisome proliferator-activated receptor $\gamma$ activators, such as pioglitazone, might suppress adverse cardiac remodeling and susceptibility to atrial fibrillation [81] but can also cause salt retention and might predispose to development of congestive heart failure.

\section{Pharmacological Rate Control}

Commonly, beta-blockers, nondihydropyridine calcium channel blockers, and digitalis are appropriate for most patients with persistent or permanent atrial fibrillation for whom control of ventricular rate is desired. For most patients, a target heart rate of 60 to 80 beats per minute at rest and 90 to 115 beats per minute during moderate exercise is appropriate.

For the AFFIRM study, adequate control was defined as an average heart rate of up to $80 \mathrm{bpm}$ at rest and either an average rate of up to $100 \mathrm{bpm}$ over at least 18 hours of ambulatory Holter monitoring with no rate greater than $100 \%$ of the maximum age-adjusted predicted exercise heart rate, or a maximum heart rate of $110 \mathrm{bpm}$ during a 6-minute walk test [37].

The selection of appropriate rate-control therapy for each patient should include consideration of the drug's potential impact on comorbid conditions such as hypertension, ischemic heart disease, and hypertrophic cardiomyopathy [36].

Generally, beta-blockers and nondihydropyridine calcium channel blockers are well tolerated; however, they are not always effective at controlling heart rate [82].

Beta-blockers maybe especially useful in the presence of high adrenergic tone or symptomatic myocardial ischemia occurring in association with AF, but they should be used with caution in patients with asthma $[6,59]$. Amiodarone, usually initiated for rhythm control, may continue to be used inadvertently for rate control when patients have lapsed into permanent $\mathrm{AF}[6,13]$. Long-term use of amiodarone may result in end-organ toxicity (pulmonary, hepatic, thyroid, neurologic, and skin) [83].

Digitalics are effective for control of heart rate at rest but not during exercise. The patients should be monitored for signs of digoxin toxicity, especially in those with reduced renal function, advanced age, acute or chronic hypoxia, or thyroid disease $[6,84]$.

Nondihydropyridine calcium channel antagonists should be avoided in patients with systolic heart failure because of their negative inotropic effect [6].

According to latest ESC guidelines, dronedarone is a firstline drug for rhythm control in patient with AF, but it is not currently approved for permanent AF to pharmacological rate control [6]. 


\section{Antithrombotic Management}

Unless contraindicated, chronic oral anticoagulation therapy $(\mathrm{OAC})$ is recommended in patients with a CHADS2 (cardiac failure, hypertension, age, diabetes, and stroke (doubled)) score of $\geq 2[6,85]$ to achieve an international normalized ratio (INR) between 2.0 and 3.0. In patients with a CHADS2 score of $0-1$, or where a more detailed stroke risk assessment is indicated, the latest guidelines recommend the use of the CHA2DS2-VASc (congestive heart failure, hypertension, age $\geq 75$ (doubled), diabetes, stroke (doubled), vascular disease, age 65-74, and sex category (female)) score [86]. Indeed, in patients with CHA2DS2-VASc $\geq 2$ OAC is recommended whereas with CHA2DS2-VASc $=1$ either OAC or aspirin $75-325 \mathrm{mg}$ daily can be chosen although OAC should be preferred. In case of CHA2DS2-VASc $=0$, no antithrombotic therapy is preferred although $75-325 \mathrm{mg}$ daily aspirin can be administered depending on the physician's choice.

Moreover, the new AF guidelines emphasize the importance of bleeding risk assessment before starting anticoagulation. In this case, the HAS-BLE bleeding risk score [87] is recommended (hypertension, abnormal renal and liver function, stroke, bleeding, labile INRs, elderly ( $>65$ years), drugs, or alcohol concomitantly). A score of $\geq 3$ is considered indicative of "high-risk" patients who require caution and regular review after starting antithrombotic therapy [6]. Multiple studies have demonstrated that oral anticoagulation with warfarin is effective for prevention of thromboembolism in AF patients [88-93], but it is underused because of the risk of bleeding [94]. Dabigatran etexilate is a new, effective, reversible, rapid-acting, oral direct inhibitor of thrombin [95]. Dabigatran has been shown to be at least as safe and effective as warfarin therapy in RE-LY, a large-scale, international, multicenter trial (randomized evaluation of long-term anticoagulant therapy) [96]. Where oral anticoagulation is an appropriate therapy, dabigatran may be considered as an alternative to adjusted -dose vitamin $\mathrm{K}$ antagonist (VKA) therapy. If a patient is at low risk of bleeding (e.g., HAS-BLED score of 02), dabigatran $150 \mathrm{mg}$ b.i.d. may be considered in view of the improved efficacy in the prevention of stroke and systemic embolism (but lower rates of intracranial haemorrhage and similar rates of major bleeding events, when compared with warfarin).

When a patient has a measurable risk of bleeding (e.g., HAS-BLED score of $\geq 3$ ), dabigatran etexilate $110 \mathrm{mg}$ b.i.d. may be considered, in view of a similar efficacy in the prevention of stroke and systemic embolism (but lower rates of intracranial hemorrhage and of major bleeding compared with VKA). In patients with CHA2DS2-VASc score $\geq 2$, dabigatran $110 \mathrm{mg}$ b.i.d. may be considered, in view of a similar efficacy with VKA in the prevention of stroke and systemic embolism but lower rates of intracranial haemorrhage and major bleeding compared with the VKA and aspirin [6].

In patients with no stroke risk factors (e.g., CHA2DS2VASc $=0$ ), either aspirin $75-325 \mathrm{mg}$ daily or no antithrombotic therapy is recommended. Where possible, no antithrombotic therapy should be considered for such patients, given the limited data on the benefits of aspirin in this patient group (i.e., lone $\mathrm{AF}$ ) and the potential for adverse effects, especially bleeding [6].

The RE-LY [75] was reviewed by the 2011 Focused Update Writing Group [7], but recommendations about its use are not included in this focused update, because dabigatran was not approved for clinical use by the FDA at the time of organizational approval.

\section{Conclusions}

Although effective therapies have been identified in concrete cases, a treatment modality offering efficacy in most cases of $\mathrm{AF}$ remains to be established. It is essential to gain further insight to the physiopathological mechanisms of $\mathrm{AF}$ to develop drugs with improved efficacy and safety profiles in the treatment of this widespread cardiac arrhythmia.

\section{Acknowledgment}

The authors gratefully acknowledge Dr. Judith Wilson for the English revision of the paper.

\section{References}

[1] W. B. Kannel, R. D. Abbott, D. D. Savage, and P. M. McNamara, "Epidemiologic features of chronic atrial fibrillation. The Framingham study," New England Journal of Medicine, vol. 306, no. 17, pp. 1018-1022, 1982.

[2] A. S. Go, E. M. Hylek, K. A. Phillips et al., "Prevalence of diagnosed atrial fibrillation in adults: national implications for rhythm management and stroke prevention: the anticoagulation and risk factors in atrial fibrillation (ATRIA) study," Journal of the American Medical Association, vol. 285, no. 18, pp. 2370-2375, 2001.

[3] W. M. Feinberg, J. L. Blackshear, A. Laupacis, R. Kronmal, and R. G. Hart, "Prevalence, age distribution, and gender of patients with atrial fibrillation: analysis and implications," Archives of Internal Medicine, vol. 155, no. 5, pp. 469-473, 1995.

[4] S. Stewart, C. L. Hart, D. J. Hole, and J. J. V. McMurray, "A population-based study of the long-term risks associated with atrial fibrillation: 20-year follow-up of the Renfrew/Paisley study," American Journal of Medicine, vol. 113, no. 5, pp. 359364, 2002.

[5] W. B. Kannel and E. J. Benjamin, "Status of the epidemiology of atrial fibrillation," Medical Clinics of North America, vol. 92, no. 1, pp. 17-40, 2008.

[6] A. J. Camm, P. Kirchhof, G. Y. H. Lip et al., "Guidelines for the management of atrial fibrillation," European Heart Journal, vol. 31, no. 19, pp. 2369-2429, 2010.

[7] L. S. Wann, A. B. Curtis, K. A. Ellenbogen et al., "2011 ACCF/AHA/HRS focused update on the management of patients with atrial fibrillation (update on dabigatran): a report of the American College of Cardiology Foundation/American Heart Association Task Force on Practice Guidelines," Heart Rhythm, vol. 8, no. 1, pp. 157-176, 2011.

[8] D. Dobrev and S. Nattel, "New antiarrhythmic drugs for treatment of atrial fibrillation," The Lancet, vol. 375, no. 9721, pp. 1212-1223, 2010. 
[9] S. Nattel and L. Carlsson, "Innovative approaches to antiarrhythmic drug therapy," Nature Reviews Drug Discovery, vol. 5, no. 12, pp. 1034-1049, 2006.

[10] S. Nattel and L. H. Opie, "Controversies in atrial fibrillation," Lancet, vol. 367, no. 9506, pp. 262-272, 2006.

[11] M. J. Riley and N. F. Marrouche, "Ablation of atrial fibrillation," Current Problems in Cardiology, vol. 31, no. 5, pp. 361390, 2006.

[12] G. B. Forleo, L. Santini, and F. Romeo, "Present concepts in managementof atrial fibrillation. From drug therapy to ablation," World Journal of Cardiology, vol. 1, no. 1, pp. 11-22, 2009.

[13] V. Fuster, L. E. Rydén, D. S. Cannom et al., "ACC/AHA/ESC 2006 guidelines for the management of patients with atrial fibrillation: fulltext: a report of the American College of Cardiology/ American Heart Association Task Force on practice guidelines and the European Society of Cardiology Committee for Practice Guidelines (Writing Committee to Revise the 2001 Guidelines for the Management of Patients With Atrial Fibrillation)," Europace, vol. 8, no. 9, pp. 651-745, 2006.

[14] H. J. G. M. Crijns, P. den Heijer, L. M. Van Wijk, and K. I. Lie, "Successful use of flecainide in atrial fibrillation with rapid ventricular rate in the Wolff-Parkinson-White syndrome," American Heart Journal, vol. 115, no. 6, pp. 1317-1321, 1988.

[15] A. Capucci, G. Boriani, G. L. Botto et al., "Conversion of recent-onset atrial fibrillation by a single oral loading dose of Propafenone or Flecainide," American Journal of Cardiology, vol. 74, no. 5, pp. 503-505, 1994.

[16] G. Boriani, M. Biffi, A. Capucci et al., "Oral propafenone to convert recent-onset atrial fibrillation in patients with and without underlying heart disease: a randomized, controlled trial," Annals of Internal Medicine, vol. 126, no. 8, pp. 621-625, 1997.

[17] S. Nattel, P. Khairy, D. Roy et al., "New approaches to atrial fibrillation management: a critical review of a rapidly evolving field," Drugs, vol. 62, no. 16, pp. 2377-2397, 2002.

[18] B. N. Singh, "Amiodarone as paradigm for developing new drugs for atrial fibrillation," Journal of Cardiovascular Pharmacology, vol. 52, no. 4, pp. 300-305, 2008.

[19] R. H. Foster, M. I. Wilde, and A. Markham, "Ibutilide. A review of its pharmacological properties and clinical potential in the acute management of atrial flutter and fibrillation," Drugs, vol. 54, no. 2, pp. 312-330, 1997.

[20] K. A. Ellenbogen, B. S. Stambler, M. A. Wood et al., "Efficacy of intravenous ibutilide for rapid termination of atrial fibrillation and atrial flutter: a dose-response study," Journal of the American College of Cardiology, vol. 28, no. 1, pp. 130-136, 1996.

[21] B. S. Stambler, M. A. Wood, K. A. Ellenbogen, K. T. Perry, L. K. Wakefield, and J. T. VanderLugt, "Efficacy and safety of repeated intravenous doses of ibutilide for rapid conversion of atrial flutter or fibrillation," Circulation, vol. 94, no. 7, pp. 1613-1621, 1996.

[22] M. A. Nabih, P. Prcevski, B. S. Fromm et al., "Effect of ibutilide, a new Class III agent, on sustained atrial fibrillation in a canine model of acute ischemia and myocardial dysfunction induced by microembolization," PACE, vol. 16, no. 10, pp. 1975-1983, 1993.

[23] M. G. Cimini, M. N. Brunden, and J. K. Gibson, "Effects of ibutilide fumarate, a novel antiarrhythmic agent, and its enantiomers on isolated rabbit myocardium," European Journal of Pharmacology, vol. 222, no. 1, pp. 93-98, 1992.

[24] K. S. Lee, "Ibutilide, a new compound with potent class III antiarrhythmic activity, activates a slow inward $\mathrm{Na}^{+}$current in guinea pig ventricular cells," Journal of Pharmacology and Experimental Therapeutics, vol. 262, no. 1, pp. 99-108, 1992.

[25] T. Yang, D. J. Snyders, and D. M. Roden, "Ibutilide, a methanesulfonanilide antiarrhythmic, is a potent blocker of the rapidly activating delayed rectifier $\mathrm{K}$ current $(\mathrm{I}(\mathrm{Kr}))$ in AT-1 cells: concentration-, time-, voltage-, and use-dependent effects," Circulation, vol. 91, no. 6, pp. 1799-1806, 1995.

[26] K. S. Lee, T. D. Tsai, and E. W. Lee, "Membrane activity of class III antiarrhythmic compounds; a comparison between ibutilide, d-sotalol, E-4031, sematilide and dofetilide," European Journal of Pharmacology, vol. 234, no. 1, pp. 43-53, 1993.

[27] I. G. Stiell, G. Dickinson, N. N. Butterfield et al., "Vernakalant hydrochloride: a novel atrial-selective agent for the cardioversionof recent-onset atrial fibrillation in the emergency department," Academic Emergency Medicine, vol. 17, no. 11, pp. 1175-1182, 2010.

[28] P. R. Kowey, P. Dorian, L. B. Mitchell et al., "Vernakalant hydrochloride for the rapid conversion of atrial fibrillation after cardiac surgery: a randomized, double-blind, placebocontrolled trial," Circulation: Arrhythmia and Electrophysiology, vol. 2, no. 6, pp. 652-659, 2009.

[29] D. Roy, C. M. Pratt, C. Torp-Pedersen et al., "Vernakalant hydrochloride for rapid conversion of atrial fibrillation: a phase 3, randomized, placebo-controlled trial," Circulation, vol. 117, no. 12, pp. 1518-1525, 2008.

[30] D. Fedida, P. M. R. Orth, J. Y. C. Chen et al., "The mechanism of atrial antiarrhythmic action of RSD1235," Journal of Cardiovascular Electrophysiology, vol. 16, no. 11, pp. 12271238, 2005.

[31] D. Roy, B. H. Rowe, I. G. Stiell et al., "A randomized, controlled trial of RSD1235, a novel anti-arrhythmic agent, in the treatment of recent onset atrial fibrillation," Journal of the American College of Cardiology, vol. 44, no. 12, pp. 2355-2361, 2004.

[32] European Medicines Agency (EMA), "European Public Assessment Report - Brinavess," February 2011, http://www .ema.europa.eu/docs/en_GB/document_library/EPAR_-_Public_assessment_report/human/001215/WC500097150.pdf.

[33] D. Tian and W. H. Frishman, "Vernakalant: a new drug to treat patients with acute onset atrial fibrillation," Cardiology in Review, vol. 19, no. 1, pp. 41-44, 2011.

[34] A. J. Camm, A. Capucci, S. H. Hohnloser et al., "A randomized active-controlled study comparing the efficacy and safety of vernakalant to amiodarone in recent-onset atrial fibrillation," Journal of the American College of Cardiology, vol. 57, no. 3, pp. 313-321, 2011.

[35] P. Alboni, G. L. Botto, N. Baldi et al., "Outpatient treatment of recent-onset atrial fibrillation with the "pill-in-the-pocket" approach," New England Journal of Medicine, vol. 351, no. 23, pp. 2384-2391, 2004.

[36] K. A. Eagle, D. S. Cannom, and D. A. Garcia, "Management of atrial fibrillation: translating clinical trial data into clinical practice," American Journal of Medicine, vol. 124, no. 1, pp. 414, 2011.

[37] B. Olshansky, L. E. Rosenfeld, A. L. Warner et al., "The Atrial Fibrillation Follow-upInvestigation of Rhythm Management (AFFIRM) study: approaches to control rate in atrial fibrillation," Journal of the American College of Cardiology, vol. 43, no. 7, pp. 1201-1208, 2004.

[38] G. Opolski, A. Torbicki, D. A. Kosior et al., "Rate control vs rhythm control in patients with nonvalvular persistent atrial fibrillation: the results of the Polish how to treat chronic atrial fibrillation (HOT CAFE) study," Chest, vol. 126, no. 2, pp. 476486, 2004. 
[39] S. H. Hohnloser, K.-H. Kuck, and J. Lilienthal, "Rhythm or rate control in atrial fibrillation-pharmacological intervention in atrial fibrillation (PIAF): a randomised trial," Lancet, vol. 356, no. 9244, pp. 1789-1794, 2000.

[40] I. C. Van Gelder, V. E. Hagens, H. A. Bosker et al., "A comparison of rate control and rhythm control in patients with recurrent persistent atrial fibrillation," New England Journal of Medicine, vol. 347, no. 23, pp. 1834-1840, 2002.

[41] J. Carlsson, S. Miketic, J. Windeler et al., "Randomized trial of rate-control versus rhythm-control in persistent atrial fibrillation: the strategies of treatment of atrial fibrillation (STAF) study," Journal of the American College of Cardiology, vol. 41, no. 10, pp. 1690-1696, 2003.

[42] D. Roy, M. Talajic, S. Nattel et al., "Rhythm control versus rate control for atrial fibrillation and heart failure," New England Journal of Medicine, vol. 358, no. 25, pp. 2667-2677, 2008.

[43] J. A. Reiffel, "Atrial fibrillation: what have recent trials taught us regarding pharmacologic management of rate and rhythm control?" PACE, vol. 34, no. 2, pp. 247-259, 2011.

[44] S. Kathofer, D. Thomas, and C. A. Karle, "The novel antiarrhythmic drug dronedarone: comparison with amiodarone," Cardiovascular Drug Reviews, vol. 23, no. 3, pp. 217-230, 2005.

[45] K. M. Dale and C. M. White, "Dronedarone: an anniodarone analog for the treatment of atrial fibrillation and atrial flutter," Annals of Pharmacotherapy, vol. 41, no. 4, pp. 599-605, 2007.

[46] European Medicines Agency, January 2011, http://www.ema .europa.eu/docs/en_GB/document_library/EPAR_-_Summary_for_the_public/human/001043/WC500044536.pdf.

[47] Y. Tschuppert, T. Buclin, L. E. Rothuizen et al., "Effect of dronedarone on renal function in healthy subjects," British Journal of Clinical Pharmacology, vol. 64, no. 6, pp. 785-791, 2007.

[48] E. E. Van der Wall, "New guidelines for the management of atrial fibrillation: what's new?" Netherlands Heart Journal, vol. 18, no. 11, p. 519, 2010.

[49] S. H. Hohnloser, H. J. G. M. Crijns, M. Van Eickels et al., "Effect of dronedarone on cardiovascular events in atrial fibrillation," New England Journal of Medicine, vol. 360, no. 7, pp. 668-678, 2009.

[50] L. Køber, C. Torp-Pedersen, J. J. V. McMurray et al., "Increased mortality after dronedarone therapy for severe heart failure," New England Journal of Medicine, vol. 358, no. 25, pp. 2678 2687, 2008.

[51] B. N. Singh, S. J. Connolly, H. J. G. M. Crijns et al., "Dronedarone for maintenance of sinus rhythm in atrial fibrillation or flutter," New England Journal of Medicine, vol. 357, no. 10, pp. 987-999, 2007.

[52] P. Touboul, J. Brugada, A. Capucci, H. J. G. M. Crijns, N. Edvardsson, and S. H. Hohnloser, "Dronedarone for prevention of atrial fibrillation: a dose-ranging study," European Heart Journal, vol. 24, no. 16, pp. 1481-1487, 2003.

[53] J. -M. Davy, M. Herold, C. Hoglund et al., "Dronedarone for the control of ventricular rate in permanent atrial fibrillation: the Efficacy and safety of dRonedArone for The cOntrol of ventricular rate during atrial fibrillation (ERATO) study," American Heart Journal, vol. 156, no. 3, pp. 527.e1-527.e9, 2008.

[54] F. T. Wegener, J. R. Ehrlich, and S. H. Hohnloser, "Dronedarone: an emerging agent with rhythm- and rate-controlling effects," Journal of Cardiovascular Electrophysiology, vol. 17, no. 2, pp. S17-S20, 2006.

[55] J. Y. Le Heuzey, G. M. De Ferrari, D. Radzik, M. Santini, J. Zhu, and J. M. Davy, "A short-term, randomized, doubleblind, parallel-group study to evaluate the efficacy and safety of dronedarone versus amiodarone in patients with persistent atrial fibrillation: the dionysos study," Journal of Cardiovascular Electrophysiology, vol. 21, no. 6, pp. 597-605, 2010.

[56] L. Køber, C. Torp-Pedersen, J. J. V. McMurray et al., "Increased mortality after dronedarone therapy for severe heart failure," New England Journal of Medicine, vol. 358, no. 25, pp. 26782687, 2008.

[57] S. H. Hohnloser, H. J. G. M. Crijns, M. Van Eickels et al., "Effect of dronedarone on cardiovascular events in atrial fibrillation," New England Journal of Medicine, vol. 360, no. 7, pp. 668-678, 2009.

[58] R. L. Page, S. J. Connolly, H. J. Crijns et al., "Rhythm- and rate-controlling effects of dronedarone in patients with atrial fibrillation: insights from the ATHENA trial," Circulation, vol. 118: 827, abstract 4097, 2008.

[59] C. Patel, G. X. Yan, and P. R. Kowey, "Dronedarone," Circulation, vol. 120, no. 7, pp. 636-644, 2009.

[60] B. N. Singh, "Amiodarone as paradigm for developing new drugs for atrial fibrillation," Journal of Cardiovascular Pharmacology, vol. 52, no. 4, pp. 300-305, 2008.

[61] N. Gassanov, M. C. Brandt, G. Michels, M. Lindner, F. Er, and U. C. Hoppe, "Angiotensin II-induced changes of calcium sparks and ionic currents in human atrial myocytes: potential role for early remodeling in atrial fibrillation," Cell Calcium, vol. 39, no. 2, pp. 175-186, 2006.

[62] K. K. Griendling, C. A. Minieri, J. D. Ollerenshaw, and R. W. Alexander, "Angiotensin II stimulates NADH and NADPH oxidase activity in cultured vascular smooth muscle cells," Circulation Research, vol. 74, no. 6, pp. 1141-1148, 1994.

[63] J. R. Ehrlich, S. H. Hohnloser, and S. Mattel, "Role of angiotensin system and effects of its inhibition in atrial fibrillation: clinical and experimental evidence," European Heart Journal, vol. 27, no. 5, pp. 512-518, 2006.

[64] E. Vermes, J. C. Tardif, M. G. Bourassa et al., "Enalapril decreases the incidence of atrial fibrillation in patients with left ventricular dysfunction: insight from the studies of left ventricular dysfunction (SOLVD) trials," Circulation, vol. 107, no. 23, pp. 2926-2931, 2003.

[65] A. H. Madrid, C. Escobar, J. M. G. Rebollo et al., "Angiotensin receptor blocker as adjunctive therapy for rhythm control in atrial fibrillation: results of the irbesartan-amiodarone trial," Cardiac Electrophysiology Review, vol. 7, no. 3, pp. 243-246, 2003.

[66] Q. Ji, Y. Mei, X. Wang et al., "Combination of irbesartan and amiodarone to maintain sinus rhythm in patients with persistent atrial fibrillation after rheumatic valve replacement," Circulation Journal, vol. 74, no. 9, pp. 1873-1879, 2010.

[67] M. Disertori, R. Latini, S. Barlera et al., "Valsartan for prevention of recurrent atrial fibrillation," New England Journal of Medicine, vol. 360, no. 16, pp. 1606-1617, 2009.

[68] European Society of Cardiology, "Irbesartan reduces heart failure in patients with quivering heart," Sepember 2009, http://www.escardio.org/congresses/esc-2009/news/Pages/ ACTIVE-I-trial.aspx.

[69] Y. Sun, F. J. A. Ramires, and K. T. Weber, "Fibrosis of atria and great vessels in response to angiotensin II or aldosterone infusion," Cardiovascular Research, vol. 35, no. 1, pp. 138-147, 1997.

[70] S. C. Shroff, K. Ryu, N. L. Martovitz, B. D. Hoit, and B. S. Stambler, "Selective aldosterone blockade suppresses atrial tachyarrhythmias in heart failure," Journal of Cardiovascular Electrophysiology, vol. 17, no. 5, pp. 534-541, 2006. 
[71] U. Dixen, L. Ravn, C. Soeby-Rasmussen et al., "Raised plasma aldosterone and natriuretic peptides in atrial fibrillation," Cardiology, vol. 108, no. 1, pp. 35-39, 2007.

[72] D. A. Pei, L. Li, Z. Y. Xu et al., "Expression of mineralocorticoid receptor and 11-beta-hydroxysteroid dehydrogenase type 2 in human atria during chronic atrial fibrillation: study of 25 cases," National Medical Journal of China, vol. 87, no. 12, pp. 816-819, 2007.

[73] P. Milliez, N. DeAngelis, C. Rucker-Martin et al., "Spironolactone reduces fibrosis of dilated atria during heart failure in rats with myocardial infarction," European Heart Journal, vol. 26, no. 20, pp. 2193-2199, 2005.

[74] A. Shiroshita-Takeshita, B. J. J. M. Brundel, J. Lavoie, and S. Nattel, "Prednisone prevents atrial fibrillation promotion by atrial tachycardia remodeling in dogs," Cardiovascular Research, vol. 69, no. 4, pp. 865-875, 2006.

[75] J. Dernellis and M. Panaretou, "Relationship between Creactive protein concentrations during glucocorticoid therapy and recurrent atrial fibrillation," European Heart Journal, vol. 25, no. 13, pp. 1100-1107, 2004.

[76] A. Shiroshita-Takeshita, G. Schram, J. Lavoie, and S. Nattel, "Effect of simvastatin and antioxidant vitamins on atrial fibrillation promotion by atrial-tachycardia remodeling in dogs," Circulation, vol. 110, no. 16, pp. 2313-2319, 2004.

[77] A. Shiroshita-Takeshita, B. J. J. M. Brundel, B. Burstein et al., "Effects of simvastatin on the development of the atrial fibrillation substrate in dogs with congestive heart failure," Cardiovascular Research, vol. 74, no. 1, pp. 75-84, 2007.

[78] O. Adam, H. R. Neuberger, M. Böhm, and U. Laufs, "Prevention of atrial fibrillation with 3-hydroxy-3-methylglutaryl coenzyme A reductase inhibitors," Circulation, vol. 118, no. 12, pp. 1285-1293, 2008.

[79] I. Savelieva and J. Camm, "Statins and polyunsaturated fatty acids for treatment of atrial fibrillation," Nature Clinical Practice Cardiovascular Medicine, vol. 5, no. 1, pp. 30-41, 2008.

[80] M. Sakabe, A. Shiroshita-Takeshita, A. Maguy et al., "Omega3 polyunsaturated fatty acids prevent atrial fibrillation associated with heart failure but not atrial tachycardia remodeling," Circulation, vol. 116, no. 19, pp. 2101-2109, 2007.

[81] M. Shimano, Y. Tsuji, Y. Inden et al., "Pioglitazone, a peroxisome proliferator-activated receptor-gamma activator, attenuates atrial fibrosis and atrial fibrillation promotion in rabbits with congestive heart failure," Heart Rhythm, vol. 5, no. 3, pp. 451-459, 2008.

[82] I. C. Van Gelder, D. G. Wyse, M. L. Chandler et al., "Does intensity of rate-control influence outcome in atrial fibrillation? An analysis of pooled data from the RACE and AFFIRM studies," Europace, vol. 8, no. 11, pp. 935-942, 2006.

[83] V. R. Vorperian, T. C. Havighurst, S. Miller, and C. T. January, "Adverse effects of low dose amiodarone: a meta-analysis," Journal of the American College of Cardiology, vol. 30, no. 3, pp. 791-801, 1997.

[84] J. P. Di Marco, "Adenosine and digoxin," in Cardiac Electrophysiology: From Cell to Bedside, D. P. Zipes and J. Jalife, Eds., pp. 942-949, W.B. Saunders, Philadelphia, Pa, USA, 4th edition, 2004.

[85] A. S. Go, E. M. Hylek, Y. Chang et al., "Anticoagulation therapy for stroke prevention in atrial fibrillation: how well do randomized trials translate into clinical practice?" Journal of the American Medical Association, vol. 290, no. 20, pp. 26852692, 2003.

[86] G. Y. H. Lip, R. Nieuwlaat, R. Pisters, D. A. Lane, and H. J. G. M. Crijns, "Refining clinical risk stratification for predicting stroke and thromboembolism in atrial fibrillation using a novel risk factor-based approach: the Euro Heart Survey on atrial fibrillation," Chest, vol. 137, no. 2, pp. 263-272, 2010.

[87] R. Pisters, D. A. Lane, R. Nieuwlaat, C. B. De Vos, H. J. G. M. Crijns, and G. Y. H. Lip, "A novel user-friendly score (HAS$\mathrm{BLED})$ to assess one-year risk of major bleeding in atrial fibrillation patients: the Euro Heart Survey," Chest, vol. 138, no. 5, pp. 1093-1100, 2010.

[88] D. E. Singer, R. A. Hughes, D. R. Gress et al., "The effect of low-dose warfarin on the risk of stroke in patients with nonrheumatic atrial fibrillation," New England Journal of Medicine, vol. 323, no. 22, pp. 1505-1511, 1990.

[89] R. McBride, "Stroke prevention in atrial fibrillation study: final results," Circulation, vol. 84, no. 2, pp. 527-539, 1991.

[90] J. C. Van Latum, P. C. Vermeulen, A. Den Ouden et al., "Secondary prevention in non-rheumatic atrial fibrillation after transient ischaemic attack or minor stroke," Lancet, vol. 342, no. 8882, pp. 1255-1262, 1993.

[91] P. Petersen, J. Godtfredsen, B. Andersen, G. Boysen, and E. D. Andersen, "Placebo-controlled, randomised trial of warfarin and aspirin for prevention of thromboembolic complications in chronic atrial fibrillation. The Copenhagen AFASAK study," Lancet, vol. 1, no. 8631, pp. 175-179, 1989.

[92] M. D. Ezekowitz, S. L. Bridgers, K. E. James et al., "Warfarin in the prevention of stroke associated with nonrheumatic atrial fibrillation," New England Journal of Medicine, vol. 327, no. 20, pp. 1406-1412, 1992.

[93] S. J. Connolly, A. Laupacis, M. Gent et al., "Canadian Atrial Fibrillation Anticoagulation (CAFA) Study," Journal of the American College of Cardiology, vol. 18, no. 2, pp. 349-355, 1991.

[94] R. G. Hart, L. A. Pearce, and M. I. Aguilar, "Meta-analysis: antithrombotic therapy to prevent stroke in patients who have nonvalvular atrial fibrillation," Annals of Internal Medicine, vol. 146, no. 12, pp. 857-867, 2007.

[95] F. M. Siddiqui and A. I. Qureshi, "Dabigatran etexilate, a new oral direct thrombin inhibitor, for stroke prevention in patients with atrial fibrillation," Expert Opinion on Pharmacotherapy, vol. 11, no. 8, pp. 1403-1411, 2010.

[96] S. J. Connolly, M. D. Ezekowitz, S. Yusuf et al., "Dabigatran versus warfarin in patients with atrial fibrillation," New England Journal of Medicine, vol. 361, no. 12, pp. 1139-1151, 2009. 


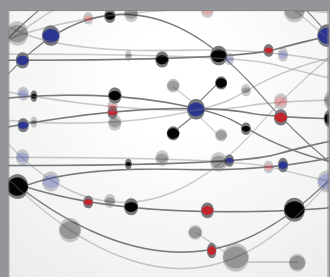

The Scientific World Journal
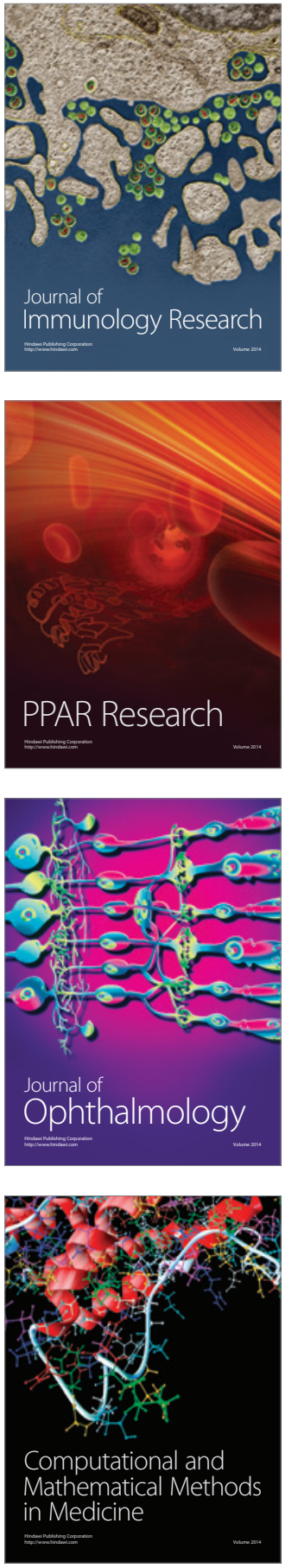

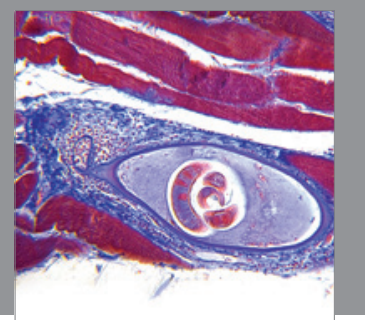

Gastroenterology

Research and Practice
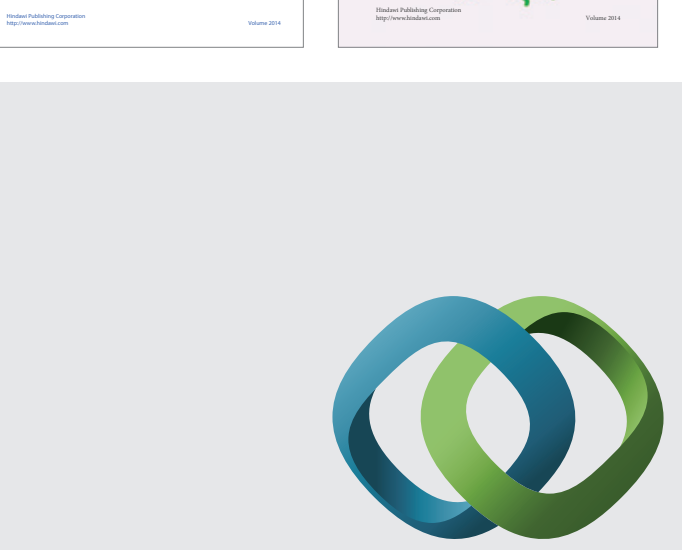

\section{Hindawi}

Submit your manuscripts at

http://www.hindawi.com
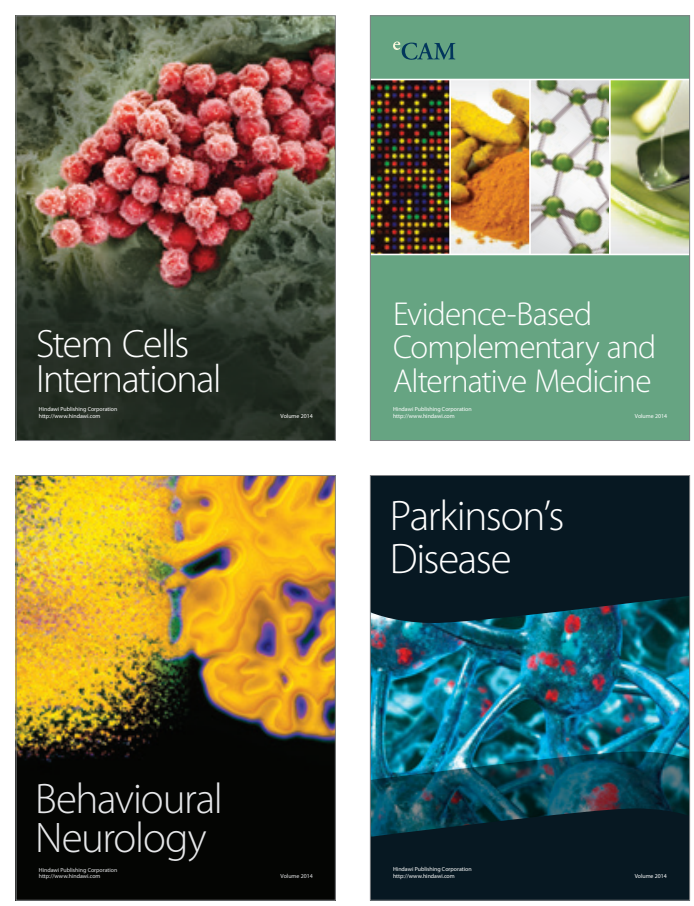

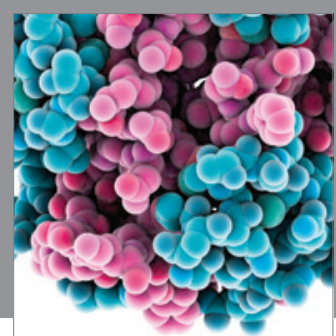

Journal of
Diabetes Research

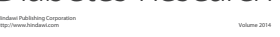

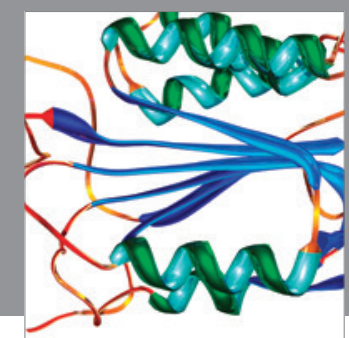

Disease Markers
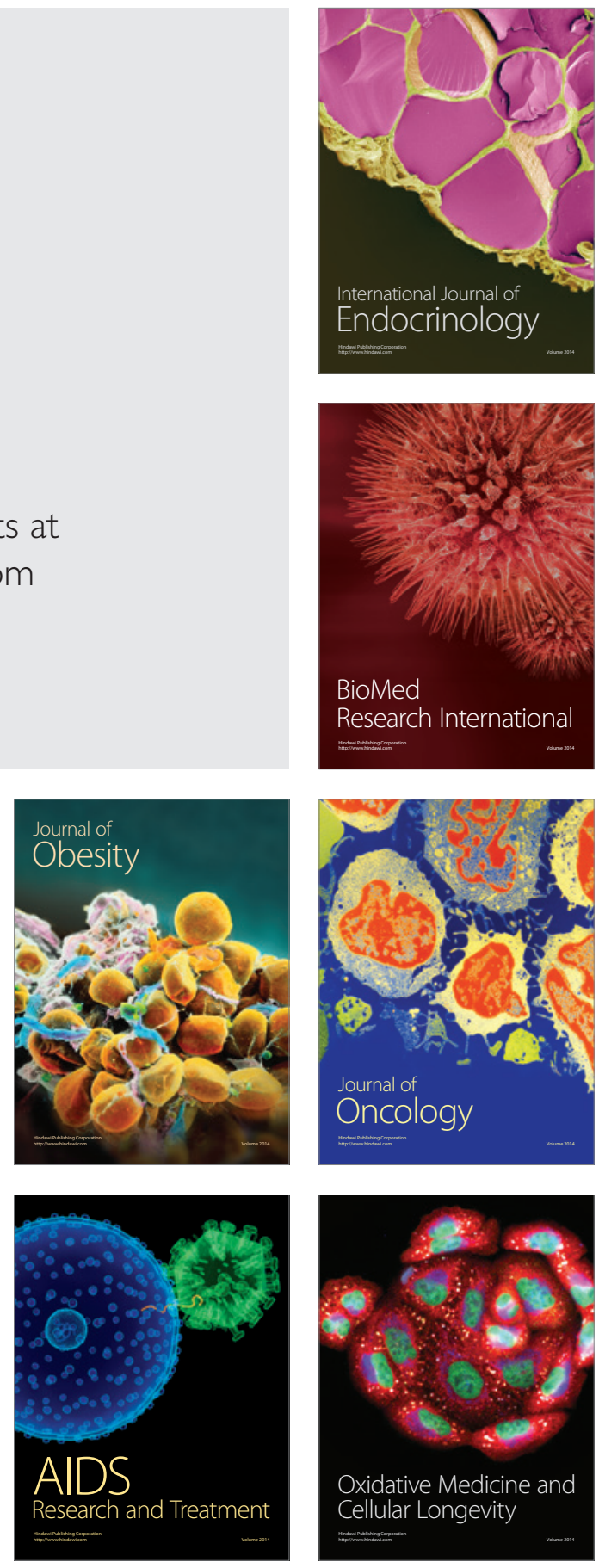